\title{
PDE Observer for All-Solid-State Batteries via an Electrochemical Model
}

\author{
Dong Zhang ${ }^{1}$, Shu-Xia Tang ${ }^{2}$, Luis D. Couto ${ }^{3}$, and Venkatasubramanian Viswanathan ${ }^{1}$
}

\begin{abstract}
All-solid-state batteries are one of the most promising candidates for next-generation energy storage devices capable of delivering high specific energy. Significant effort has been spent on understanding the degradation mechanisms associated with dendrite formation, while energy management and model-based estimation/control for solid-state batteries has received very limited attention. This paper examines a partial differential equation (PDE) state estimation scheme for a onedimensional electrochemical all-solid-state battery model, using voltage and current measurements only. The state estimation framework exploits the active disturbance rejection control and PDE backstepping techniques, and we rigorously prove estimation error system stability. Electrochemical model-based estimator based on PDE models identifies physical variables for all-solid-state batteries, thus enables high-fidelity monitoring and optimal control in future battery management systems.
\end{abstract}

\section{INTRODUCTION}

Lithium-ion (Li-ion) batteries have revolutionized modern life by enabling electric vehicles and portable power electronics. However, safety concerns are increasing in the quest for pushing the performance envelope, leading to unpredictable circumstances such as overcharging and abuses [1], [2]. Electrification of other sectors such as long-haul trucks [3] and aviation [4] requires even higher specific energy while also delivering on other metrics, e.g. specific power and cost.

Among all next-generation battery chemistries, all-solidstate batteries with a metallic lithium anode offers a significant increase in specific energy as well as possibly better thermal stability [5]. Many practical issues related to uncontrollable lithium dendrite growth [6] and poor cycling efficiency [7] still linger but are being addressed and mitigated [8]. The general working principles of solid-state lithium metal batteries are similar to that of conventional $\mathrm{Li}$ ion batteries. A major distinction is that the porous electrode theory in Li-ion batteries [9] is no longer necessary for lithium metal anode as it involves plating and stripping of metallic lithium instead of intercalation and de-intercalation.

The majority of research in battery modeling and control has been centered around conventional $\mathrm{Li}$-ion batteries with porous composite electrodes [10], [11], [12], [13], and very few efforts have been dedicated to solid-state

\footnotetext{
${ }^{1}$ Dong Zhang and Venkatasubramanian Viswanathan are with Department of Mechanical Engineering, Carnegie Mellon University, Pittsburgh, PA 15312, USA \{dongzhr, venkvis\} e cmu.edu

${ }^{2}$ Shu-Xia Tang, the corresponding author, is with Department of Mechanical Engineering, Texas Tech University, Lubbock, TX 79409, USA shuxia.tangettu. edu

${ }^{3}$ Luis D. Couto is with Department of Control Engineering and System Analysis, Université Libre de Bruxelles, B-1050 Brussels, Belgium lcoutome@ulb.ac.be. He would like to thank the Wiener-Anspach Foundation for its financial support.
}

batteries. Danilov et al. developed a one-dimensional model to simulate the performance of a $\mathrm{Li}\left|\mathrm{Li}_{3} \mathrm{PO}_{4}\right| \mathrm{LiCoO}_{2}$ cell [14], accounting for charge transfer kinetics, lithium diffusion in electrode, and diffusion and migration of ions in solid electrolyte. Following [14], Kazemi et al. enhances modeling accuracy at high current by incorporating phase-transition processes and time-varying diffusion coefficient [15]. Fabre et al. presented a model for $\mathrm{Li}|\mathrm{LiPON}| \mathrm{LiCoO}_{2}$ solid-state thin-film microbattery, along with several electrochemical experiments used to carry out accurate characterization of model parameters [16]. Behrou et al. advances solid-state battery modeling via a finite element framework for electrochemical and mechanical interactions with damage evolution [17]. Phase-field modeling has been used to understand lithium metal anode morphological instabilities [6].

Nevertheless, many of the aforementioned models are intricate for real-time control purposes - a barrier often encountered with electrochemical models. Only recently has the community begun to seek control-oriented modeling and/or model reductions. For instance, Kim et al. proposed a Kalman filter (KF) for solid-state battery state of charge (SOC) estimation [18]. The model explores multiple reduction scenarios, including neglecting the generation/recombination of Li-ions in the solid electrolyte. Moreover, a reduced-order electrochemical model was proposed in [19] thanks to Padé approximation and polynomial approximations of the concentration distributions.

In this paper, we advance the research field of model-based all-solid-state battery estimation and control by developing a partial differential equation (PDE)-based state estimation framework. Multiple novel theoretical methods are created and integrated, including PDE disturbance estimator and backstepping state estimator. The model analysis and estimator designs in this paper are completely performed on the original PDE system without any prior spatial discretization, thus retaining the physical significance of the equations and of the phenomena that they represent. The stability and asymptotic convergence of the proposed estimators are mathematically guaranteed and proved, which marks one of the notable advantages against KF-based estimator designs.

\section{Solid-State Battery Modeling}

The schematic of an all-solid-state Li-ion battery is depicted in Fig. 1, which is comprised of three main elements: anode, cathode, and electrolyte. The anode consists of metallic lithium. The cathode material is of the conventional intercalation chemistry, e.g., $\mathrm{LiCoO}_{2}$. The electrodes are separated by a solid-state electrolyte, shown in this case as 


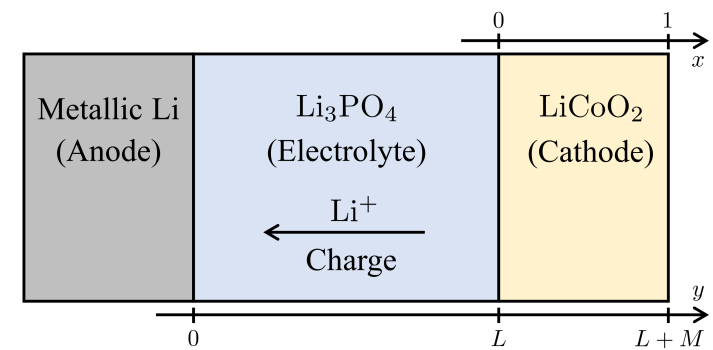

Fig. 1. A general schematic of a planar all-solid-state Li-ion battery [14].

amorphous $\mathrm{Li}_{3} \mathrm{PO}_{4}$. A one-dimensional model that describes the dynamics of the solid-state battery is adopted from [14]. It is assumed that the charge transfer kinetics are neglected in the anode since it is made of metallic lithium. A complete description of model symbols is defined in Table I.

\section{A. Solid Electrolyte}

In the electrolyte, the bound lithium, designated as $\mathrm{Li}^{0}$, is transferred into mobile $\mathrm{Li}^{+}$through the ionization reaction:

$$
\mathrm{Li}^{0} \underset{k_{d}}{\stackrel{k_{r}}{\rightleftharpoons}} \mathrm{Li}^{+}+\mathrm{n}^{-} .
$$

The conductivity of the $\mathrm{Li}_{3} \mathrm{PO}_{4}$-based solid electrolyte is induced by the transport of $\mathrm{Li}^{+}$ions. The mathematical model, describing the diffusion-migration process combined with the generation/recombination (1), can be represented by the following nonlinear reaction-diffusion PDE system:

$$
\begin{aligned}
\frac{\partial c_{\mathrm{Li}^{+}}}{\partial t}(y, t) & =\frac{2 D_{\mathrm{Li}^{+}} D_{\mathrm{n}^{-}}}{D_{\mathrm{Li}^{+}}+D_{\mathrm{n}^{-}}} \frac{\partial^{2} c_{\mathrm{Li}^{+}}}{\partial y^{2}}(y, t)+r\left(c_{\mathrm{Li}^{+}}(y, t)\right), \\
\frac{\partial c_{\mathrm{Li}^{+}}}{\partial y}(0, t) & =\frac{I(t)}{2 F A D_{\mathrm{Li}^{+}}}, \\
\frac{\partial c_{\mathrm{Li}^{+}}}{\partial y}(L, t) & =\frac{I(t)}{2 F A D_{\mathrm{Li}^{+}}},
\end{aligned}
$$

with $c_{\mathrm{Li}^{+}}(y, 0)=\delta c_{\mathrm{Li}^{+}, \max }(y)$ and $y \in[0, L]$. The charge carrier generation is given by $r\left(c_{\mathrm{Li}^{+}}(y, t)\right)=\alpha c_{\mathrm{Li}^{+}}^{2}+$ $\beta c_{\mathrm{Li}^{+}}+\gamma$, with $\alpha=-k_{r}, \beta=-k_{r} \delta^{2} c_{\mathrm{Li}^{+}, 0} /(1-\delta)$, and $\gamma=k_{r} \delta^{2} c_{\mathrm{Li}^{+}, 0} /(1-\delta)$. The total mass-transfer overpotential across the solid electrolyte, which includes the diffusion and migration overpotentials, can then be expressed by

$$
\eta_{\mathrm{mt}}=\frac{R T}{F} \ln \left[\frac{c_{\mathrm{Li}^{+}}(L, t)}{c_{\mathrm{Li}^{+}}(0, t)}\right]-\int_{0}^{L} E(y, t) \mathrm{d} y,
$$

where the analytic expression for the electric field is

$$
\begin{aligned}
E(y, t)= & \frac{R T}{F} \frac{1}{c_{\mathrm{Li}^{+}}(y, t)}\left(-\frac{I(t)}{2 F A D_{\mathrm{Li}^{+}}}\right. \\
& \left.+\frac{D_{\mathrm{Li}^{+}}-D_{\mathrm{n}^{-}}}{D_{\mathrm{Li}^{+}}+D_{\mathrm{n}^{-}}}\left[\frac{\partial c_{\mathrm{Li}^{+}}(y, t)}{\partial y}-\frac{I(t)}{2 F A D_{\mathrm{Li}^{+}}}\right]\right) .
\end{aligned}
$$

\section{B. Cathode}

\begin{tabular}{|c|c|c|c|}
\hline Symbols & Description & Units & Values \\
\hline$A$ & Cell cross sectional area & {$\left[\mathrm{m}^{2}\right]$} & $1 \times 10^{-4}$ \\
\hline$c_{\mathrm{Li}^{+}}$ & $\mathrm{Li}^{+}$conc. in electrolyte & {$\left[\mathrm{mol} / \mathrm{m}^{3}\right]$} & {$[-]$} \\
\hline$c_{\mathrm{Li}^{+}, \text {max }}$ & 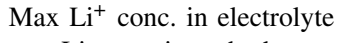 & {$\left[\mathrm{mol} / \mathrm{m}^{3}\right]$} & $6.01 \times 10^{4}$ \\
\hline$c_{\text {Lis }}$ & $\mathrm{Li}$ conc. in cathode & {$\left[\mathrm{mol} / \mathrm{m}^{3}\right]$} & {$[-]$} \\
\hline$c_{\text {Lis,max }}$ & Max $\mathrm{Li}^{+}$conc. in cathode & {$\left[\mathrm{mol} / \mathrm{m}^{3}\right]$} & $2.33 \times 10^{4}$ \\
\hline$D_{\mathrm{Li}^{+}}$ & $\mathrm{Li}^{+}$Diffusivity in electrolyte & {$\left[\mathrm{m}^{2} / \mathrm{s}\right]$} & $1.76 \times 10^{-15}$ \\
\hline$D_{\mathrm{n}^{-}}$ & $\mathrm{n}^{-}$Diffusivity in electrolyte & {$\left[\mathrm{m}^{2} / \mathrm{s}\right]$} & $2.1 \times 10^{-15}$ \\
\hline$D_{\text {Lis }}$ & Li Diffusivity in cathode & {$\left[\mathrm{m}^{2} / \mathrm{s}\right]$} & $0.9 \times 10^{-15}$ \\
\hline$F$ & Faraday's constant & {$[\mathrm{C} / \mathrm{mol}]$} & 96485 \\
\hline$I$ & Applied current & {$[\mathrm{A}]$} & {$[-]$} \\
\hline$k_{r}$ & Recombination reaction rate & {$\left[\mathrm{m}^{3} / \mathrm{mol}-\mathrm{s}\right]$} & $9 \times 10^{-9}$ \\
\hline$k_{0}^{\text {app }}$ & Apparent rate constant & {$\left[\mathrm{m}^{3} / \mathrm{mol}-\mathrm{s}\right]$} & $5.1 \times 10^{-4}$ \\
\hline$M$ & Cathode thickness & {$[\mathrm{m}]$} & $3.2 \times 10^{-7}$ \\
\hline$L$ & Solid electrolyte thickness & {$[\mathrm{m}]$} & $1.5 \times 10^{-6}$ \\
\hline$R$ & Universal gas constant & {$[\mathrm{J} / \mathrm{mol}-\mathrm{K}]$} & 8.314 \\
\hline$T$ & Temperature & {$[\mathrm{K}]$} & 298 \\
\hline$t$ & Time & {$[\mathrm{s}]$} & {$[-]$} \\
\hline$\delta$ & $\begin{array}{l}\text { Fraction of mobile } \mathrm{Li}^{+} \\
\text {in electrolyte in equilibrium }\end{array}$ & {$[-]$} & 0.18 \\
\hline$\eta_{\mathrm{mt}}$ & $\begin{array}{l}\text { Mass-transfer overpotential } \\
\text { across the electrolyte }\end{array}$ & [V] & {$[-]$} \\
\hline$\eta_{\mathrm{ct}}, \eta_{\mathrm{d}}$ & $\begin{array}{l}\text { Charge transfer/Diffusion } \\
\text { overpotential in cathode }\end{array}$ & [V] & {$[-]$} \\
\hline
\end{tabular}

The electrochemical charge transfer reactions at the cathode can be represented by

$$
\mathrm{LiCoO}_{2} \rightleftharpoons \mathrm{Li}_{1-x} \mathrm{CoO}_{2}+x \mathrm{Li}^{+}+x \mathrm{e}^{-} \quad(0 \leq x \leq 0.5) \text {. }
$$

TABLE I

SOlid STATE BATTERy MOdel Symbol Description

Assuming the migration process can be neglected and $\mathrm{Li}^{+}$ diffusion due to concentration gradient is the primary means of charge transfer [18], the mass transport of Li-ions inside the cathode is governed by the Fick's law of diffusion,

$$
\begin{aligned}
\frac{\partial c_{\text {Lis }}}{\partial t}(y, t) & =D_{\text {Lis }} \frac{\partial^{2} c_{\text {Lis }}}{\partial y^{2}}(y, t), \\
\frac{\partial c_{\text {Lis }}}{\partial y}(L, t) & =\frac{I(t)}{F A D_{\text {Lis }}}, \\
\frac{\partial c_{\text {Lis }}}{\partial y}(L+M, t) & =0,
\end{aligned}
$$

with $c_{\text {Lis }}(y, 0)=c_{\text {Lis }, 0}(y)$. The Butler-Volmer kinetics links the charge transfer overpotential and the applied current via

$$
I(t)=i_{0}(t)\left(e^{\frac{\alpha_{a} F \eta_{\mathrm{ct}}}{R T}}-e^{\frac{-\alpha_{c} F \eta_{\mathrm{ct}}}{R T}}\right),
$$

where the exchange current density $i_{0}(t)$ reads [16]

$$
\begin{aligned}
i_{0}(t)= & F\left[k_{a}\left(c_{\mathrm{Li}^{+}, \max }-c_{\mathrm{Li}^{+}}(L, t)\right) c_{\mathrm{Lis}}(L, t)\right]^{\alpha_{c}} \\
& \times\left[k_{c}\left(c_{\mathrm{Lis}, \max }-c_{\mathrm{Lis}}(L, t)\right) c_{\mathrm{Li}^{+}}(L, t)\right]^{\alpha_{a}} .
\end{aligned}
$$

Let $\theta$ be the normalized Li concentration in the cathode, i.e., $\theta=c_{\text {Lis }} / c_{\text {Lis,max }}$. The diffusion overpotential is computed according to [14], [20],

$$
\eta_{d}=E_{\mathrm{eq}}\left(\theta_{s}(t)\right)-E_{\mathrm{eq}}(\bar{\theta}(t)),
$$

in which $E_{\text {eq }}$ denotes the equilibrium potential for the cathode, whereas $\theta_{s}(t)=\theta(L, t)$ and $\bar{\theta}(t)$ is the normalized volume-averaged (bulk) $\mathrm{Li}$ concentration in the cathode. Empirical functions of $E_{\text {eq }}$ can be obtained via experimental curve fitting [16], and thermodynamically consistent opencircuit curves may be found through the Redlish-Kister formalism [21]. 


\section{Output Voltage}

The total overpotential is represented as

$$
\eta_{t}=\eta_{\mathrm{mt}}+\eta_{\mathrm{ct}}+\eta_{\mathrm{d}}
$$

where the respective terms were presented in (5), (11), and (13). Ultimately, the output voltage is the combined effect from equilibrium voltage and total overpotential,

$$
V(t)=E_{\mathrm{eq}}(\bar{\theta})+\eta_{t}=\eta_{\mathrm{mt}}+\eta_{\mathrm{ct}}+E_{\mathrm{eq}}\left(\theta_{s}\right) .
$$

\section{Model Reduction}

Assumption 1: $\mathrm{Li}^{+}$concentration across the solid electrolyte is uniform [16], [18].

Evidently, the contribution of $\eta_{\mathrm{mt}}$ to the total overpotential $\eta_{t}$ is negligible in the relatively high SOC end [18]. More importantly, this assumption allows us to reduce the size and complexity of the model towards enabling real-time estimation and control tasks. Ultimately, the dynamics (2)-(4) are hereafter neglected. However, we introduce the following model compensation and normalization:

i. The modeling error from Assumption 1 is compensated by a boundary disturbance in the cathode dynamics. Namely, an unknown disturbance is artificially injected at the cathode-electrolyte interface (9), to account for the neglected electrolyte dynamics.

ii. Since the dynamics within solid electrolyte is ignored, we now re-position the origin of the spatial coordinate to be at the cathode-electrolyte interface.

iii. We perform normalization by scaling spatial and temporal coordinates as $x=y / M, \bar{t}=D_{\text {Lis }} t / M^{2}$ (Fig. 1).

Henceforth we will drop the bar over the temporal coordinate to simplify notation when the context is clear. Consequently, these operations transform the cathode dynamics (8)-(10) into the following PDE with Neumann boundary conditions:

$$
\begin{aligned}
& u_{t}(x, t)=u_{x x}(x, t), \\
& u_{x}(0, t)=U(t)+d(t), \\
& u_{x}(1, t)=0,
\end{aligned}
$$

with $u(x, 0)=u_{0}(x)$. Herein, $u_{t}=\partial u / \partial t, u_{x x}=\partial^{2} u / \partial x^{2}$, and $u=c_{\text {Lis. }}$. Further, $U(t)=I(t) \cdot M /\left(F A D_{\text {Lis }}\right)$ is the boundary control. The term $d(t)$ is a boundary disturbance used to compensate the loss of electrolyte dynamics. By assuming $\alpha_{a}=\alpha_{c}=0.5$ [18], [22], the output function after the model reduction can be computed via the nonlinear map

$$
V_{r}(t)=\eta_{\mathrm{ct}}+E_{\mathrm{eq}}\left(\theta_{s}\right)=\frac{2 R T}{F} \sinh ^{-1}\left[\frac{I(t)}{2 i_{0}(t)}\right]+E_{\mathrm{eq}}\left(\theta_{s}\right),
$$

where $\theta_{s}(t)=u(0, t) / c_{\text {Lis,max }}$. Since lithium concentration is assumed uniform across the solid electrolyte, exchange current density can be simplified to

$$
i_{0}(t)=F k_{0}^{\mathrm{app}}[u(0, t)]^{\alpha_{c}}\left[c_{\text {Lis, } \max }-u(0, t)\right]^{\alpha_{a}},
$$

where $k_{0}^{\text {app }}$ is an apparent rate constant for the cathode.

Remark 1: It is expected that the lost contributions to the voltage after neglecting electrolyte dynamics will be indirectly recompensed by the boundary disturbance $d(t)$ in (17) at the cathode-electrolyte interface in the reducedorder model. We will later design a disturbance estimator to estimate the unknown disturbance $d(t)$, such that the voltage response of the reduced-order model (16)-(20) matches the voltage of the original full-order model.

\section{Disturbance And State Estimators}

The objective of this section is to develop a state estimator for the lithium concentration profile in the cathode based on (i) the reduced-order model (16)-(20) and (ii) voltage of the full-order model (1)-(15). The main steps of the estimator design are (i) inverting the output function (19) to obtain "boundary measurement", (ii) estimating the unknown boundary disturbance $d(t)$ in order to compensate the loss of electrolyte dynamics, and finally (iii) estimating state $u(x, t)$ with knowledge of the disturbance estimate.

\section{A. Output Function Inversion}

To implement a boundary disturbance/state estimator, we need $\theta_{s}(t)$, or $u(0, t)$. Nonetheless, $\theta_{s}(t)$ is not directly measured. The only measurable signal (voltage) is related to $\theta_{s}(t)$ via the nonlinear map (19). As such, we develop a gradient algorithm to process $\theta_{s}(t)$ from voltage measurement $V(t)$ via the inversion of the output function (19) [22].

First, re-write the output function (19) as $V_{r}=h\left(\theta_{s}, t\right)$. Next, denote $\hat{\theta}_{s}(t)$ the outcome from the output function inversion. Then the error between the true and the inverted version is $\tilde{\theta}_{S}(t)=\theta_{s}(t)-\hat{\theta}_{s}(t)$. With that, re-write

$$
V_{r}(t)=h\left(\tilde{\theta}_{s}+\hat{\theta}_{s}, t\right) \text {. }
$$

Then, we perform first-order Taylor series approximation with respect to $\tilde{\theta}_{s}$ around $\tilde{\theta}_{s}=0$ to get

$$
V_{r}(t) \approx h\left(\hat{\theta}_{s}, t\right)+\frac{\partial h}{\partial \theta_{s}}\left(\hat{\theta}_{s}, t\right) \cdot \tilde{\theta}_{s} .
$$

The approximated output function in the form (22) can be re-expressed as $e(t)=\phi(t) \tilde{\theta}_{s}$ where

$$
e(t)=V_{r}(t)-h\left(\hat{\theta}_{s}, t\right), \quad \phi(t)=\frac{\partial h}{\partial \theta_{s}}\left(\hat{\theta}_{s}, t\right) .
$$

The regressor $\phi(t)$ depends upon the inversion $\hat{\theta}_{s}(t)$, and $e(t)$ is additionally determined by the measured signal. We adopt the recursive least-squares algorithm with forgetting factor to update $\hat{\theta}_{s}(t)$ [23, Chapter 4]:

$$
\begin{aligned}
\dot{\hat{\theta}}_{s}(t) & =P \epsilon(t) \phi(t), \quad \hat{\theta}_{s}(0)=\hat{\theta}_{s}^{0}, \\
\dot{P}(t) & =\beta P(t)-P(t) \frac{\phi(t) \phi^{\top}(t)}{m^{2}(t)} P(t), \quad P(0)=P_{0}, \\
\epsilon(t) & =\frac{e(t)-\phi(t) \hat{\theta}_{s}(t)}{m^{2}(t)}, \\
m^{2}(t) & =1+c \phi^{\top}(t) \phi(t), \quad c>0 .
\end{aligned}
$$

Hereafter, $\theta_{s}(t)$ (whose numerical value is given by $\hat{\theta}_{s}(t)$ ) is regarded as the "pseudo-measurement" of the plant model dynamics (16)-(18). That is, $y(t)=u(0, t)$.

Remark 2: We have essentially produced an estimate $\hat{\theta}_{S}$ for the boundary value $\theta_{s}$ that is consistent with the measured (full-order) voltage $V(t)$ through the output function (19) in the reduced-order model. 


\section{B. Disturbance Estimator Design}

In this section, we detail the disturbance estimator design [24] using the boundary measurement $y(t)$. The objective of the disturbance estimator is to construct a $\hat{d}(t)$, which asymptotically estimates $d(t)$ in the boundary condition (17), such that system (16)-(18) generates a $\theta_{s}(t)$ that is equal to $\hat{\theta}_{s}(t)$. In this way, the neglected voltage component resulting from dropping the electrolyte dynamics is fully compensated.

We introduce the following auxiliary system,

$$
\begin{aligned}
v_{t}(x, t) & =v_{x x}(x, t), \\
v(0, t) & =y(t), \\
v_{x}(1, t) & =0 .
\end{aligned}
$$

$v$-system consists of a copy of the plant model dynamics (16), and is driven by $y(t)$. Let the estimate for $d(t)$ be

$$
\hat{d}(t)=v_{x}(0, t)-U(t) .
$$

System (28)-(31) forms the disturbance estimator. Prior to proving the convergence, we present the following lemma.

Lemma 1: For any function $f(x, t)$ with $x \in[0,1]$ and $t \in[0, \infty)$, that is three times continuously differentiable on $x \in[0,1]$, we have

$$
\left\|f_{x x}(x, t)\right\|^{2} \leq 2 f_{x x}^{2}(0, t)+4\left\|f_{x x x}(x, t)\right\|^{2},
$$

where $\|\cdot\|$ denotes the $L_{2}$ norm.

Theorem 1: The disturbance estimate $\hat{d}(t)$ given by (31) exponentially converges to $d(t)$. Namely, $\lim _{t \rightarrow \infty} \tilde{d}(t)=0$, where $\tilde{d}(t)=d(t)-\hat{d}(t)$.

Proof: Let $w(x, t)=u(x, t)-v(x, t)$, which satisfies

$$
\begin{aligned}
w_{t}(x, t) & =w_{x x}(x, t), \\
w(0, t) & =0, \\
w_{x}(1, t) & =0 .
\end{aligned}
$$

Consider the Lyapunov functional

$$
\Omega(t)=\frac{1}{2} \int_{0}^{1} w^{2} \mathrm{~d} x+\frac{1}{2} \int_{0}^{1} w_{x}^{2} \mathrm{~d} x+\frac{1}{2} \int_{0}^{1} w_{x x}^{2} \mathrm{~d} x .
$$

The time derivative of $\Omega(t)$ is then computed by applying integral by parts,

$$
\begin{aligned}
\dot{\Omega}= & \left.w(x) w_{x}(x)\right|_{0} ^{1}-\left\|w_{x}\right\|^{2}+\left.w_{x}(x) w_{t}(x)\right|_{0} ^{1}-\int_{0}^{1} w_{t} w_{x x} \mathrm{~d} x \\
& +\left.w_{x x}(x) w_{x t}(x)\right|_{0} ^{1}-\int_{0}^{1} w_{x t} w_{x x x} \mathrm{~d} x .
\end{aligned}
$$

Since $w_{t}(0)=w_{x t}(1)=w_{x t}(0)=0$, we can conclude

$$
\begin{aligned}
\dot{\Omega} & =-\left\|w_{x}\right\|^{2}-\left\|w_{x x}\right\|^{2}-\left\|w_{x x x}\right\|^{2} \\
& \leq-\frac{1}{4}\|w\|^{2}-\frac{1}{4}\left\|w_{x}\right\|^{2}-\frac{1}{4}\left\|w_{x x}\right\|^{2} \leq-\frac{1}{2} \Omega,
\end{aligned}
$$

where Lemma 1 has been utilized. From (38), the exponential stability of $\Omega(t)$ indicates that $\|w\|,\left\|w_{x}\right\|,\left\|w_{x x}\right\| \rightarrow 0$ when $t \rightarrow \infty$. Moreover, according to the fundamental theorem of calculus and Cauchy-Schwarz Inequality,

$$
w_{x}(0, t)=w_{x}(1, t)-\int_{0}^{1} w_{x x} \mathrm{~d} x \leq\left\|w_{x x}\right\| \rightarrow 0
$$

as $t \rightarrow \infty$. A direct computation shows that

$$
w_{x}(0, t)=u_{x}(0, t)-v_{x}(0, t)=d(t)-\left[v_{x}(0, t)-U(t)\right] .
$$

We can regard $\hat{d}(t)=v_{x}(0, t)-U(t)$ as the estimate of $d(t)$.

\section{State Estimator Design}

We now present a state estimator leveraging the disturbance estimation to reconstruct the lithium concentration in the cathode. The estimator is designed by using a copy of the plant model (16)-(18) with an output error injection:

$$
\begin{aligned}
& \hat{u}_{t}(x, t)=\hat{u}_{x x}(x, t)+k(x)[u(0, t)-\hat{u}(0, t)], \\
& \hat{u}_{x}(0, t)=U(t)+\hat{d}(t)+k_{1}[u(0, t)-\hat{u}(0, t)], \\
& \hat{u}_{x}(1, t)=0,
\end{aligned}
$$

with $\hat{u}(x, 0)=\hat{u}_{0}(x) \neq u_{0}(x) . \hat{u}(x, t)$ represents the estimate of $u(x, t)$, and $k(x)$ and $k_{1}$ are, respectively, spatiallydistributed and constant observer gains to be determined to guarantee the stability of estimation error $\tilde{u}(x, t)=u(x, t)-$ $\hat{u}(x, t)$. Note that the disturbance estimate $\hat{d}(t)$ is injected into the boundary of the state estimator (42), and it has been previously established that $\tilde{d}(t)=d(t)-\hat{d}(t) \rightarrow 0$ as $t \rightarrow \infty$.

Subtracting (41)-(43) from (16)-(18) yields the state estimation error dynamics,

$$
\begin{aligned}
\tilde{u}_{t}(x, t) & =\tilde{u}_{x x}(x, t)-k(x) \tilde{u}(0, t), \\
\tilde{u}_{x}(0, t) & =\tilde{d}(t)-k_{1} \tilde{u}(0, t), \\
\tilde{u}_{x}(1, t) & =0 .
\end{aligned}
$$

To determine the observer gains $k(x)$ and $k_{1}$, we adopt the PDE backstepping approach [25]. We seek a linear Volterra transformation that transforms the state of $\tilde{u}$ system to a target state $\tilde{\omega}$ by making use of the following expression,

$$
\tilde{\omega}(x, t)=\tilde{u}(x, t)-\int_{0}^{x} K(x, z) \tilde{u}(z, t) \mathrm{d} z,
$$

where $K(x, z)$ is the gain kernel to be designed. The transformation (47) maps $\tilde{u}$-system to the target system

$$
\begin{aligned}
\tilde{\omega}_{t}(x, t)= & \tilde{\omega}_{x x}(x, t)+\lambda \omega(x, t)+K(x, 0) \tilde{\omega}_{x}(0, t) \\
& +K(x, 0) K(0,0) \tilde{\omega}(0, t), \\
\tilde{\omega}_{x}(0, t)= & -q \tilde{\omega}(0, t)+\tilde{d}(t), \\
\tilde{\omega}_{x}(1, t)= & 0,
\end{aligned}
$$

in which the parameters $\lambda$ and $q$ are to be determined to ensure stability of $\tilde{\omega}$-system. To establish kernel function $K(x, z)$, we differentiate the transformation (47) with respect to $x$ and $t$. This yields the kernel PDE

$$
\begin{aligned}
K_{x x}(x, z)-K_{z z}(x, z) & =\lambda K(x, z), \\
K_{x}(1, z) & =0, \\
K(x, x) & =\frac{\lambda}{2}(x-1),
\end{aligned}
$$

whose closed-form solution is given by

$$
K(x, z)=-\lambda(1-z) \frac{J_{1}(\sqrt{\lambda(2-x-z)(x-z)})}{\sqrt{\lambda(2-x-z)(x-z)}} .
$$


where $J_{1}(\cdot)$ is the first-order Bessel Function of the first kind. From the comparison of the target system (48)-(50) with the original error system (44)-(46), it follows that the observer gain functions should be chosen to satisfy

$$
\int_{0}^{x} k(\zeta) \mathrm{d} \zeta-k(x)=K_{z}(x, 0), \quad k_{1}=q-K(0,0) .
$$

A closed-form solution of $k(x)$ from (55) is given by

$$
k(x)=-K_{z}(x, 0)-e^{x} \int_{0}^{x} e^{-\tau} K_{z}(\tau, 0) \mathrm{d} \tau .
$$

Further, it can also be proven that the linear Volterra transformation (47) is invertible [25].

Theorem 2: Let $k(x)$ and $k_{1}$ be given by (54)-(56). Suppose $\lambda$ in (48) and $q$ in (49) are chosen to satisfy

$$
q \leq-\frac{1}{2 \gamma}-\frac{k_{1}^{2}}{2 m}-\frac{\pi^{2}}{4}, \text { and } \lambda<\frac{\pi^{2}}{4}-\frac{p+m}{2} \kappa,
$$

where $\gamma, m, p$, and $\kappa$ are user-defined parameters. Since the disturbance estimate $\hat{d}(t)$ converges to $d(t)$ asymptotically (Theorem 1), the equilibrium of the target system (48)(50) is asymptotically stable in the sense of $L_{2}$ norm, i.e., $\lim _{t \rightarrow \infty}\|\tilde{\omega}\|=0$.

Proof: Consider the Lyapunov functional

$$
W(t)=\frac{1}{2} \int_{0}^{1} \tilde{\omega}^{2}(x, t) \mathrm{d} x=\frac{1}{2}\|\tilde{\omega}\|^{2} .
$$

The time derivative of the Lyapunov functional $W(t)$ along the trajectory of $\tilde{\omega}(x, t)$ is computed as

$$
\begin{aligned}
\dot{W} & =\int_{0}^{1} \tilde{\omega} \tilde{\omega}_{x x} \mathrm{~d} x+\lambda \int_{0}^{1} \omega^{2} \mathrm{~d} x+\tilde{\omega}_{x}(0) \int_{0}^{1} K(x, 0) \tilde{\omega} \mathrm{d} x \\
& +K(0,0) \tilde{\omega}(0) \int_{0}^{1} K(x, 0) \tilde{\omega} \mathrm{d} x \\
& =q \tilde{\omega}^{2}(0)-\tilde{d}(t) \tilde{\omega}(0)-\int_{0}^{1} \tilde{\omega}_{x}^{2} \mathrm{~d} x+\lambda \int_{0}^{1} \omega^{2} \mathrm{~d} x \\
& +\tilde{d}(t) \int_{0}^{1} K(x, 0) \tilde{\omega} \mathrm{d} x+k_{1} \tilde{\omega}(0) \int_{0}^{1} K(x, 0) \tilde{\omega} \mathrm{d} x .
\end{aligned}
$$

Now, imposing Young's inequality on the second, fifth, and sixth term of (59) results in

$$
\begin{aligned}
\dot{W} \leq & q \tilde{\omega}^{2}(0)+\frac{\gamma}{2} \tilde{d}^{2}+\frac{1}{2 \gamma} \tilde{\omega}^{2}(0)-\left\|\tilde{\omega}_{x}\right\|^{2}+\lambda\|\tilde{\omega}\|^{2}+\frac{1}{2 p} \tilde{d}^{2} \\
& +\frac{p+m}{2}\left(\int_{0}^{1} K(x, 0) \tilde{\omega} \mathrm{d} x\right)^{2}+\frac{k_{1}^{2}}{2 m} \tilde{\omega}^{2}(0) .
\end{aligned}
$$

Next, we apply the "variation of Wirtinger's inequality" [25], $\|\tilde{\omega}\|^{2} \leq \tilde{\omega}^{2}(0)+4\left\|\tilde{\omega}_{x}\right\|^{2} / \pi^{2}$, to the fourth term of $(60)$, and Cauchy-Schwarz inequality to the seventh term, to deduce

$$
\begin{aligned}
\dot{W} \leq & \left(q+\frac{1}{2 \gamma}+\frac{k_{1}^{2}}{2 m}+\frac{\pi^{2}}{4}\right) \tilde{\omega}^{2}(0)+\left(\frac{1}{2 p}+\frac{\gamma}{2}\right)|\tilde{d}|^{2} \\
& -\left(\frac{\pi^{2}}{4}-\lambda\right)\|\tilde{\omega}\|^{2}+\frac{p+m}{2}\|\tilde{\omega}\|^{2} \int_{0}^{1} K^{2}(x, 0) \mathrm{d} x .
\end{aligned}
$$

According to the boundedness of the Bessel Function $J_{1}(\cdot)$, or equivalently $K(x, 0)$, we have that $\int_{0}^{1} K^{2}(x, 0) \mathrm{d} x \leq \kappa$ for some $\kappa>0$. Furthermore, as per Theorem 1, for any given $D>0$, there exists a $t^{\prime} \geq 0$ such that $|\tilde{d}(t)| \leq D$ when $t \geq t^{\prime}$. Now, substituting these properties into (61),

$$
\begin{aligned}
\dot{W} \leq & \left(q+\frac{1}{2 \gamma}+\frac{k_{1}^{2}}{2 m}+\frac{\pi^{2}}{4}\right) \tilde{\omega}^{2}(0)+\left(\frac{1}{2 p}+\frac{\gamma}{2}\right) D^{2} \\
& -\left(\frac{\pi^{2}}{4}-\lambda-\frac{p+m}{2} \kappa\right)\|\tilde{\omega}\|^{2} .
\end{aligned}
$$

Let $a:=\pi^{2} / 4-\lambda-(p+m) \kappa / 2>0$ and $b:=$ $1 /(2 p)+\gamma / 2>0$. In light of (57), the above inequality (62) is simplified to $\dot{W} \leq-2 a W+b D^{2}$. This implies $\lim _{t \rightarrow \infty}\|\tilde{\omega}\| \leq \sqrt{b / a} D \rightarrow 0$, since $D$ can be arbitrarily small thanks to Theorem 1 .

Since the target system (48)-(50) is asymptotically stable and the transformation (47) is invertible, we are able to conclude that the original error system (44)-(46) is asymptotically stable: $\lim _{t \rightarrow \infty}\|\tilde{u}\|=0$.

\section{Simulation Results}

We examine the performance of the proposed estimators via simulations. The numerical study is conducted on a 10 $\mu \mathrm{Ah}$ planar thin-film all-solid-state Li-ion battery. Model parameters are retrieved from [14], [18], and are enumerated in Table I. The estimators are initialized at incorrect states to demonstrate uncertainty in initial conditions.

A $3 \mathrm{C}$ constant current discharge cycle is applied to the full-order model. Fig. 2(a) plots the applied current in terms of C-rate and the corresponding voltage response. This voltage ("measured" output) from the full-order model is then inverted based on the reduced-order voltage equation (19) as well as the output function inversion algorithm developed in Section III-A, and the outcome of the inversion is illustrated in Fig. 2(b). The accuracy of such an inversion is further validated by processing the inverted signal $\hat{\theta}_{s}(t)$ through the voltage equation (19) in reduced-order model, and the response is compared with the measured voltage $V(t)$, which is demonstrated in Fig. 2(c). Despite the initial transient period in Fig. 2(c), the root mean squared error (RMSE) between these two voltages is $0.2 \mathrm{mV}$, indicating a high accuracy from the output inversion procedure.

Next, the boundary "pseudo-measurement" $\hat{\theta}_{s}(t)$ is leveraged for the disturbance estimator, which is plotted in Fig 2(d). In particular, it is evident from Fig. 2(e) that the reduced-order model compensated by a disturbance $d(t)$ (whose estimate is $\hat{d}(t)$ ) performs strongly better than that without the disturbance, in terms of predicting the measured battery voltage response. Consequently, by taking $\lambda=1$ and $q=-10$ to satisfy (57), the state estimation error in the sense of $L_{2}$ norm is pictured in Fig. 2(f).

\section{CONClusion}

This paper reports the first PDE estimator for all-solidstate battery electrochemical models. The mathematical model towards an estimator design adopts the conventional practice of neglecting electrolyte dynamics in the solid electrolyte, yet we compensate the resultant voltage loss by 
(a). Measured Voltage \& Current

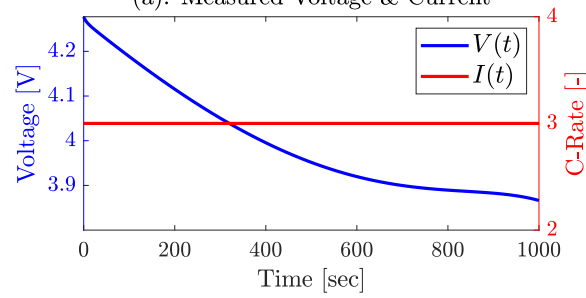

(d). Disturbance Estimator

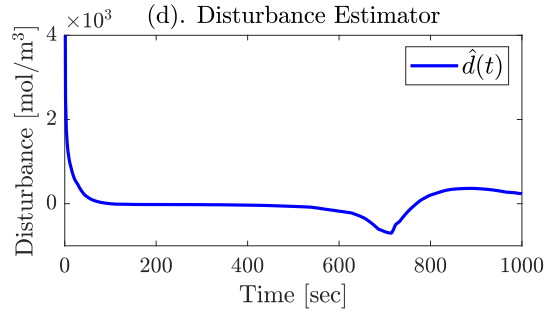

(b). Output Function Inversion

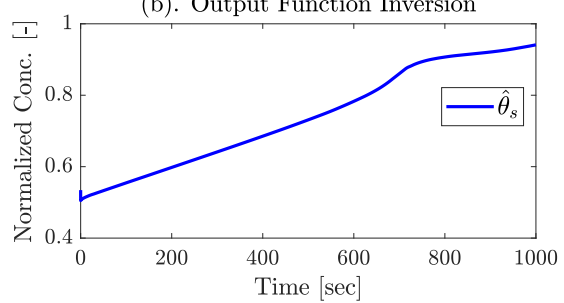

(e). Voltage Comparison

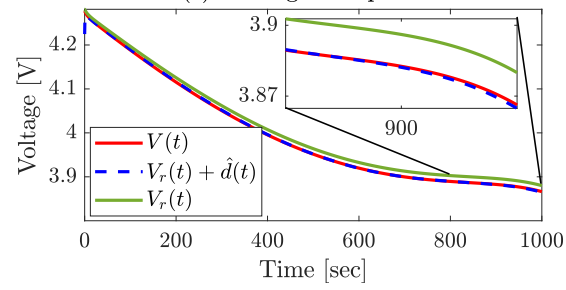

(c). Output Function Inversion Validation
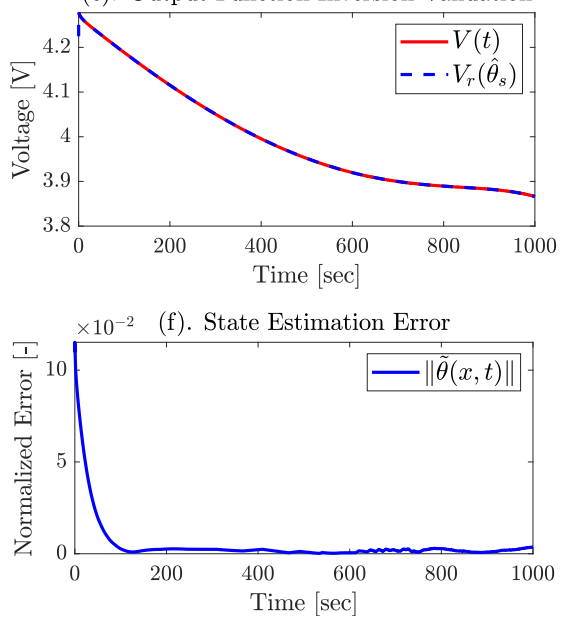

Fig. 2. State estimator results for a $3 \mathrm{C}$ constant current discharge cycle, using full-order model with electrolyte dynamics as truth model.

a boundary disturbance in the reduced-order model. We then propose a disturbance and state estimation scheme and prove convergence for the estimators for guaranteed reconstruction of lithium concentration profile. Results presented here could be viewed as a stepping stone for future advanced battery management systems for variations of all-solid-state battery.

\section{REFERENCES}

[1] Qingsong Wang, Binbin Mao, Stanislav I Stoliarov, and Jinhua Sun. A review of lithium ion battery failure mechanisms and fire prevention strategies. Progress in Energy and Combustion Science, 73:95-131, 2019.

[2] Dong Zhang, Satadru Dey, Luis D Couto, and Scott J Moura. Battery adaptive observer for a single-particle model with intercalationinduced stress. IEEE transactions on control systems technology, 2019.

[3] Shashank Sripad and Venkatasubramanian Viswanathan. Performance metrics required of next-generation batteries to make a practical electric semi truck. ACS Energy Letters, 2(7):1669-1673, 2017.

[4] Alexander Bills, Shashank Sripad, William Leif Fredericks, Madalsa Singh, and Venkatasubramanian Viswanathan. Performance metrics required of next-generation batteries to electrify commercial aircraft. ACS Energy Letters, 5(2):663-668, 2020.

[5] Paul Albertus, Susan Babinec, Scott Litzelman, and Aron Newman. Status and challenges in enabling the lithium metal electrode for highenergy and low-cost rechargeable batteries. Nature Energy, 3(1):16$21,2018$.

[6] Zijian Hong and Venkatasubramanian Viswanathan. Phase-field simulations of lithium dendrite growth with open-source software. ACS Energy Letters, 3(7):1737-1743, 2018.

[7] Xin-Bing Cheng, Rui Zhang, Chen-Zi Zhao, and Qiang Zhang. Toward safe lithium metal anode in rechargeable batteries: a review. Chemical reviews, 117(15):10403-10473, 2017.

[8] Chengyin Fu, Victor Venturi, Jinsoo Kim, Zeeshan Ahmad, Andrew W Ells, Venkatasubramanian Viswanathan, and Brett A Helms. Universal chemomechanical design rules for solid-ion conductors to prevent dendrite formation in lithium metal batteries. Nature materials, 19(7):758-766, 2020.

[9] Marc Doyle, Thomas F Fuller, and John Newman. Modeling of galvanostatic charge and discharge of the lithium/polymer/insertion cell. Journal of the Electrochemical society, 140(6):1526, 1993.

[10] Kandler A Smith, Christopher D Rahn, and Chao-Yang Wang. Control oriented 1D electrochemical model of lithium ion battery. Energy Conversion and management, 48(9):2565-2578, 2007.

[11] Dong Zhang, Satadru Dey, and Scott J Moura. Lithium-ion battery state estimation for a single particle model with intercalation-induced stress. In 2018 Annual American Control Conference (ACC), pages 2294-2299. IEEE, 2018.
[12] Domenico Di Domenico, Anna Stefanopoulou, and Giovanni Fiengo. Lithium-ion battery state of charge and critical surface charge estimation using an electrochemical model-based extended kalman filter. Journal of dynamic systems, measurement, and control, 132(6), 2010.

[13] Dong Zhang, Luis D Couto, and Scott J Moura. Electrode-level state estimation in lithium-ion batteries via kalman decomposition. IEEE Control Systems Letters, 5(5):1657-1662, 2020.

[14] D Danilov, RAH Niessen, and PHL Notten. Modeling all-solid-state Li-ion batteries. Journal of the Electrochemical Society, 158(3):A215, 2010.

[15] Namdar Kazemi, Dmitri L Danilov, Lucas Haverkate, Nancy J Dudney, Sandeep Unnikrishnan, and Peter HL Notten. Modeling of all-solidstate thin-film Li-ion batteries: Accuracy improvement. Solid State Ionics, 334:111-116, 2019.

[16] Steve D Fabre, Delphine Guy-Bouyssou, Pierre Bouillon, Frédéric Le Cras, and Charles Delacourt. Charge/discharge simulation of an allsolid-state thin-film battery using a one-dimensional model. Journal of the Electrochemical Society, 159(2):A104, 2011.

[17] Reza Behrou and Kurt Maute. Numerical modeling of damage evolution phenomenon in solid-state lithium-ion batteries. Journal of The Electrochemical Society, 164(12):A2573, 2017.

[18] Youngki Kim, Xianke Lin, Armin Abbasalinejad, Sun Ung Kim, and Seung Hyun Chung. On state estimation of all solid-state batteries. Electrochimica Acta, 317:663-672, 2019.

[19] Zhongwei Deng, Xiaosong Hu, Xianke Lin, Le Xu, Jiacheng Li, and Wenchao Guo. A reduced-order electrochemical model for all-solidstate batteries. IEEE Transactions on Transportation Electrification, 2020.

[20] Ralph E White, JOM Bockris, and BE Conway. Modern aspects of electrochemistry, no. 15, 1983.

[21] Deepak K Karthikeyan, Godfrey Sikha, and Ralph E White. Thermodynamic model development for lithium intercalation electrodes. Journal of Power Sources, 185(2):1398-1407, 2008.

[22] Scott J Moura, Federico Bribiesca Argomedo, Reinhardt Klein, Anahita Mirtabatabaei, and Miroslav Krstic. Battery state estimation for a single particle model with electrolyte dynamics. IEEE Transactions on Control Systems Technology, 25(2):453-468, 2016.

[23] Petros A Ioannou and Jing Sun. Robust adaptive control. Courier Corporation, 2012.

[24] Dong Zhang, Shu-Xia Tang, and Scott J Moura. State and disturbance estimator for unstable reaction-advection-diffusion PDE with boundary disturbance. In 2019 Proceedings of the Conference on Control and its Applications, pages 67-74. SIAM, 2019.

[25] Miroslav Krstic and Andrey Smyshlyaev. Boundary control of PDEs: A course on backstepping designs. SIAM, 2008. 